\title{
Two professions against two killer diseases: the rationale, organization, and initial experience of a cardio-oncology service
}

\author{
Mateusz Tajstra', Sławomir Blamek², Ilona Skoczylas', Aleksandra Majsnerowska1', Jacek T. Niedziela', \\ Elżbieta Gadula-Gacek ${ }^{3}$, Barbara Bobek-Billewicz ${ }^{4}$, Mariusz Gąsior ${ }^{1}$, Michał Jarząb ${ }^{5}$ \\ 13 rd Department of Cardiology, Faculty of Medical Sciences in Zabrze, Medical University of Silesia, Katowice, Poland \\ 2 Department of Radiotherapy, Maria Skłodowska-Curie National Research Institute of Oncology, Gliwice Branch, Gliwice, Poland \\ 3 Outpatient Clinic, Maria Skłodowska-Curie National Research Institute of Oncology, Gliwice Branch, Gliwice, Poland \\ 4 Department of Radiology and Diagnostic Imaging, Maria Skłodowska-Curie National Research Institute of Oncology, Gliwice Branch, Gliwice, Poland \\ 5 Breast Unit, Maria Skłodowska-Curie National Research Institute of Oncology, Gliwice Branch, Gliwice, Poland
}

\section{KEY WORDS}

cancer, cardio-

-oncology,

cardiotoxicity, service

\section{EDITORIAL}

by Pardo Sanz

and Zamorano,

see $p .112$

Correspondence to: Mateusz Tajstra, MD, PhD, 3rd Department of Cardiology, Faculty of Medical Sciences in Zabrze, Medical University of Silesia, ul. Curie-Skłodowskiej 9 , 41-800 Zabrze, Poland, phone: +48323733619 , email: mateusztajstra@wp.pl Received: August 24, 2020. Revision accepted: October 29, 2020. Published online: November 4, 2020. Kardiol Pol. 2021; 79 (2): 139-146 doi:10.33963/KP.15674 Copyright by the Author(s), 2021

\section{ABSTRACT}

BACKGROUND Cardiovascular diseases are the most common factor affecting prognosis in cancer survivors. Cardio-oncology (CO) services have been developed to solve this issue. The outcomes regarding patient demographics and clinical findings are limited and the available data include CO services evaluating patients undergoing only chemotherapy as opposed to those also undergoing radiation therapy.

AIMS We aimed to show initial experiences of the CO service implemented in a tertiary oncology center. METHODS The CO service was designed to include 2 major domains, general CO and electrotherapy consultations. This observational study included patients referred to the CO service with the following data: baseline demographics, cancer type, reasons for referral, cardiac evaluation, and initial clinical outcomes. RESULTS All patients with cancer referred to our CO service between March 2016 and December 2019 were included in the study. A total of 2762 patients (77\% women) at the mean (SD) age of 62 (12) years were referred ( $63 \%$ on an out-patient basis) for general consultations. The most frequent diagnosis was breast cancer (66\%). A total of $18 \%$ of patients were referred to the CO service due to cardiovascular complications related to cancer treatment. The CO-cardiac implantable electronic device (CIED) team evaluated 652 patients ( 515 patients with CIEDs who were qualified for radiotherapy, 48 patients with CIEDs who were assessed with magnetic resonance imaging, and 89 patients with CIEDs who underwent cancer surgery). In the total of 5872 radiotherapy sessions, there were 2 harmful interactions; no other complications during magnetic resonance imaging and surgery were recorded.

CONCLUSIONS The CO-service established within the cancer center seems to be safe and feasible.

INTRODUCTION The 2 killer diseases prevail. Cancer is currently the second most common cause of death in Europe, followed by cardiovascular diseases. ${ }^{1}$ It is estimated that 23.6 million new cancer cases worldwide will be recorded by $2030 .^{2}$

The number of patients surviving cancer has been growing rapidly, mainly due to early diagnosis and development of modern and effective anticancer therapies. Similarly, a comprehensive diagnosis, primary and secondary prevention rules, intensive pharmacotherapy, and interventional procedures have improved the outcomes in cardiovascular diseases. However, in the setting of the advanced age of the population, these factors, ironically, may often lead to a situation in which the same individuals are affected by both cardiovascular diseases and cancer. Additionally, cancer and cardiovascular diseases may share the same risk factors, which is 


\section{WHAT'S NEW?}

Cancer and cardiovascular diseases are responsible for the highest rate of death in the developed countries. Moreover, the current treatment of these 2 killer diseases may have adverse interactions. Thus, an "all-hands-on-deck" approach is needed to seek new ways to anticipate and eliminate possible undesired influence. One solution could be a cooperation between the 2 professions: cardiology and oncology. A crucial step in such a cooperation should take the form of a cardio-oncology service building. This study reveals that a cardio-oncology service developed in a cancer tertiary center in cooperation with an academic cardiovascular center with various possible interactions between cancer treatment (including modern chemotherapy, radiotherapy, and surgery) and cardiovascular complications is a safe, feasible, and comprehensive tool in this campaign. Additionally, our initial experience and findings could serve as a valuable tool for those who are planning to develop a cardio-oncology service.

amplified by cardiovascular complications of adjuvant cancer treatments including chemotherapy and radiation therapy-the so called "cardiotoxicity" which can lead to premature morbidity and death in cancer survivors. ${ }^{3-6}$

This has brought about the development of a new branch of clinical care, namely, cardio-oncology (CO), a discipline based on a multidisciplinary approach to seek solutions for the prevention, monitoring, diagnosis, and treatment of the impairment of heart structures during and after the anticancer therapy.

Historically, due to potential safety concerns, MRI was contraindicated in patients with cardiac implanted electronic devices (CIEDs), such as a pacemaker or an implantable cardioverter-defibrillator (ICD). These concerns resulted from the fact that a magnetic field could potential induce CIED failure. ${ }^{8}$ At least half of patients with CIEDs are predicted to have a clinical indication for MRI during their lifetime after the device implantation, ${ }^{9}$ and patients with cancer are a significant part of this population. Valuable MRI-related diagnostics and decision-making findings are an important part of care in oncological patients.

A growing number of patients with CIEDs require an effective cancer treatment, including radiotherapy. Modern CIEDs may be potentially damaged by external factors, including radiotherapy. Lack of full awareness on the potential implications of the influence of radiation on CIEDs may lead to an unnecessary disqualification from radiotherapy (often, the only possible way to cure cancer), incorrect qualification to removal or relocatation of an existing CIED before radiotherapy, or performing radiotherapy without the necessary precautions. ${ }^{9}$

The number of patients with CIEDs presenting for surgery, including cancer surgery, is increasing. Electromagnetic interference (EMI) is frequently encountered in the operating theater environment and can adversely affect the CIEDs function. This could entail severe consequences for patients, especially those with pacemaker dependency, or for patients and / or medical staff, as a result of the activation of high-voltage interventions of an ICD (inappropriate shocks).

These 3 areas of potential cooperation between cardiologists and oncologists are usually not included in CO services and clinics; however, we strongly believe that they are of great importance. A close cooperation between cardiologists and oncologists, the implementation of dedicated protocols, appropriate device programming, and patient monitoring significantly reduced the risk of harmful interactions of MRI / radiotherapy / EMI-CIEDs and enabled safe use of MRI and radiotherapy, as well as performing surgery in patients with CIEDs. ${ }^{10,11}$

Due to the paucity of data coming from the $\mathrm{CO}$ service and consisting in a broad clinical evaluation of cancer patients including patients demographics and clinical findings, the principal aim of this paper was to share our initial experiences of the CO service established at the tertiary Maria Skłodowska-Curie National Research Institute of Oncology, Gliwice Branch, in cooperation with the 3rd Department of Cardiology, Silesian Center for Heart Diseases. Additionally, our findings may provide some guidance to those interested in developing a program between similar independent institutions.

METHODS All cancer patients referred to our CO service between March 2016 and December 2019 were included in the study. Collected data included baseline clinical demographics, cancer type, cancer therapy, CIED type and functional parameters, and reasons for referral. At the same time, we aimed to include cancer patients in general $\mathrm{CO}$ consultations (including data on the clinical assessment before, during, and after cancer therapy) and electrotherapy CO consultations (including the care of patients with CIEDs undergoing radiotherapy, MRI, and the monitoring of CIEDs before, during, and after radiotherapy, MRI, and cancer surgery). Both of these $\mathrm{CO}$ clinic branches are supported by the Third Cardiology Department in Zabrze (FIGURE 1).

General cardio-oncology consultations General cardio-oncology consultations are performed by 3 consultant cardiologists and a dedicated clinical nurse, all of whom work on a daily basis, from Monday to Friday. According to the established protocol, all newly referred individuals undergo a clinical baseline evaluation. This includes a detailed physical examination, blood tests (including cardiac biomarkers), a resting 12-lead electrocardiogram, and resting transthoracic echocardiography. Additional diagnostic workup including invasive tests (when clinically indicated) is planned and patients are referred for an in-hospital cardiovascular 


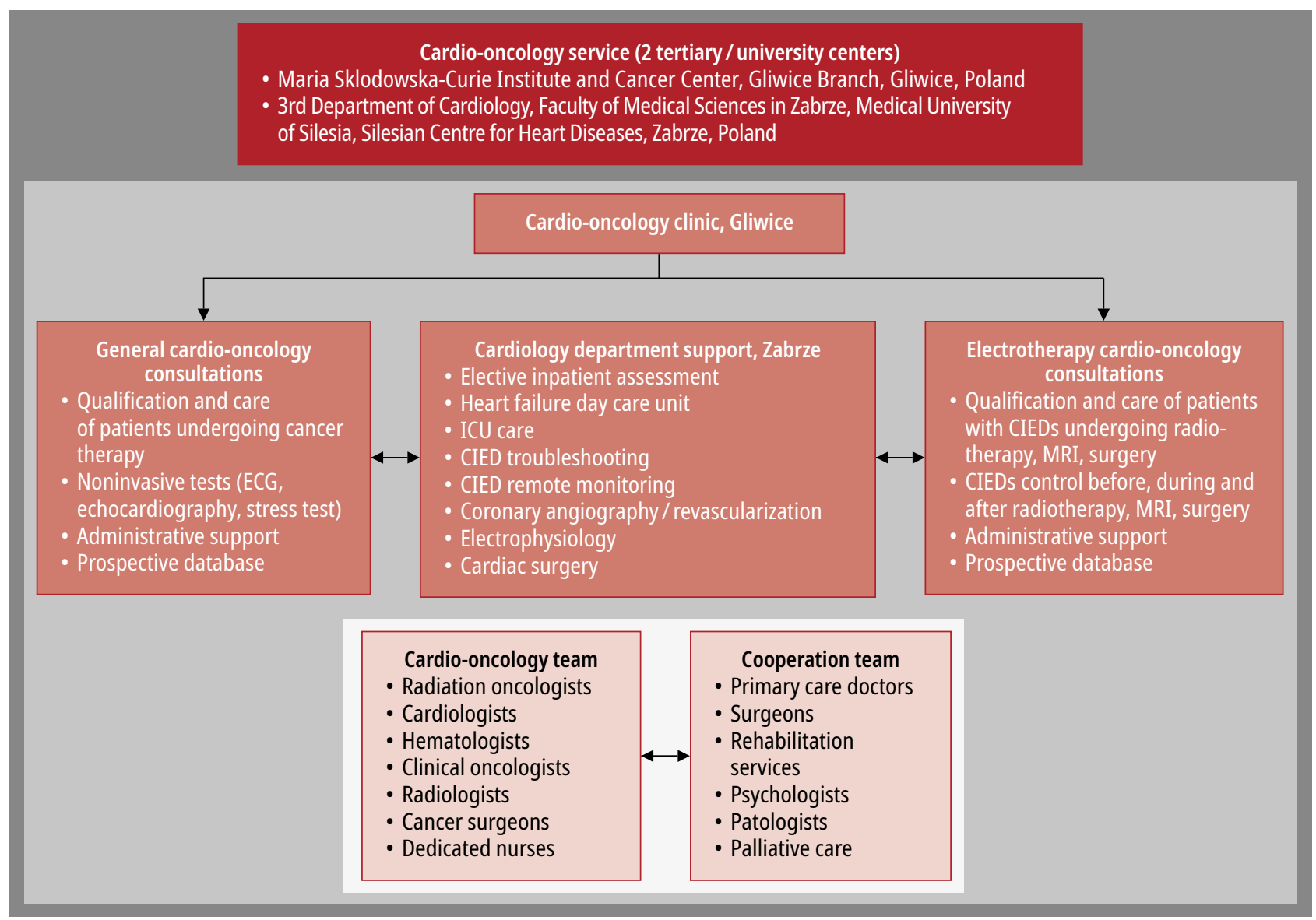

FIGURE 1 The scheme and objectives of the cardio-oncology service in Gliwice

Abbreviations: CIEDs, cardiac implantable electronic devices; ECG, electrocardiogram; ICU intensive care unit; MRI, magnetic resonance imaging

\section{TABLE 1 The main reasons for referral to the general cardio-oncology service in Gliwice}

Condition

High baseline cardiovascular risk (due to pre-existing cardiovascular diseases and / or cardiovascular risk factors, and / or a high risk of cardiovascular damages related to cancer treatment)

Asymptomatic and symptomatic cardiovascular complications related to cancer treatment (eg, left ventricle dysfunction) or related to cancer itself (pericardial effusion)

Before the intensification of cancer treatment (eg, additional drugs, a higher drug dose)

Before recruitment to a drug program

evaluation before initiating cancer treatment. TABLE 1 presents common reasons for referral to this part of the CO clinic. The clinical decision of the consultant regarding the qualification, disqualification, or temporary suspension of cancer treatment due to required additional cardiovascular optimization is based on the baseline cardiovascular risk, cancer therapy-related risk, and cancer stage assessment, following a CO team discussion of each individual case. The management protocols of modifiable cardiovascular risk factors before, during, and after cancer treatment, as well as the monitoring, prevention, and treatment of cardiovascular damage during and after the completion of cancer treatment were introduced in accordance with a number of current practice guidelines. ${ }^{12,13}$ Acute cardiac complications are also subject to treatment by the CO service. Depending on the patient's condition, he or she is treated on the spot in an oncology department of a municipal hospital or transferred to a tertiary cardiology center.

\section{Electrotherapy cardio-oncology consul-} tations At our CO service, electrotherapy cardio-oncology consultations were initially established between radiation oncologists from the Department of Radiotherapy, the Maria Skłodowska-Curie National Research Institute of Oncology, Gliwice Branch, and cardiologists from the 3rd Department of Cardiology, Silesian Center for Heart Diseases. We have initiated the cooperation to treat patients with CIEDs who were qualified for radiotherapy. 
Later, the scope of the electrotherapy CO clinic was extended to 2 additional areas: the supervision of patients with CIEDs and undergoing radiotherapy, and those qualified for MRI or for cancer surgery.

Radiotherapy in patients with cardiac implantable electronic devices In 2016, we started radiotherapy as part of the $\mathrm{CO}$ service in patients with cardiac implantable electronic devices. Initially, the supervision of patients with CIEDs followed our own protocols created on the basis of German, Dutch, and personal experiences..$^{14-17}$ The course of action was altered after the recommendations of the Heart Rhythm Society (HRS) were published. ${ }^{9}$ The service is provided in an outpatient clinic with 5 consultant cardiologists and 2 electrophysiology fellows, all trained in controlling, programming, and troubleshooting CIEDs, as well as a supervising nurse. The team operates 2 days per week based on consultations and every day if direct supervision during radiotherapy is needed. The initial consultation before radiotherapy includes a detailed examination, especially towards cardiovascular diseases and CIED control in each patient. Based on the examination and radiotherapy parameters obtained from a responsible radiation oncologist, patients are assigned to one of the 3 risk groups: low, medium, or high. Currently, depending on the result of risk-stratification, the adequate approach is based on the experts' opinion of the Heart Rhythm Association of Polish Cardiac Society and the Polish Society of Radiation Oncology. ${ }^{17}$

Magnetic resonance imaging in patients with cardiac implantable electronic devices The MRI program is performed twice a month in patients who require the MRI scan for any reason. All CIEDs are analyzed, checked, and programed in accordance with the HRS expert consensus prior to MRI and then reprogrammed and checked again after the MRI by an experienced cardiologist who is present throughout the examination.

Both MRI-conditional and non-MRI-conditional CIEDs are allowed for MRI. All parameters of the device are collected in a special form. A patient cannot be qualified for the scan in the following situations: abandoned or damaged electrodes, end of battery life status, CIED malfunctions. When abandoned or damaged electrodes are confirmed, a decision on whether or not the patient can be referred for transvenous lead extraction and then MRI is made after a discussion within the $\mathrm{CO}$ team. In our cohort, we have not had such a case so far. Electrocardiogram, blood saturation, and blood pressure are constantly monitored during the study. The study is supervised by an MRI technician, a cardiologist, and a nurse (if the intravenous contrast is required). An external defibrillator is available on site during the study. After the MRI, each patient is advised to be followed after 1 month in his or her referring center.

Cancer surgery in patients with cardiac implantable electronic devices Cancer surgery also became part of our CO service in 2018. The supervising nurse informs the consulting doctors, members of the CO-CIED team, about the list of patients scheduled for surgery. A detailed patient assessment, including CIEDs control and preoperative reprogramming (if needed), is performed a day before the surgical intervention. ${ }^{18}$ After the discharge from the surgery department, each patient is advised to be followed within 6 or 12 months in his referring center.

Statistical analysis The results for quantitative variables with a normal distribution were presented as means (SD), and for variables with a nonnormal distribution, as medians and interquartile ranges (IQR). The results for categorical variables are presented as percentage. The distribution of the continuous variables was analyzed using the Shapiro-Wilk test. The STATISTICA 10 software (StatSoft, Inc., Tulsa, Oklahoma, United States) was used for all calculations.

The study was approved by an appropriate institutional review board and-given the retrospective nature of the analysis-a written informed consent to participate in the study was not required.

RESULTS General cardio-oncology consultations Until the end of 2019, a total of 2762 general CO consultations were performed as part of the CO service. Women with breast cancer constituted the most numerous group among the referred individuals. Cardiovascular complications related to cancer treatment were recorded in $18 \%$ of patients. The number of referred patients per year, their baseline characteristics, and cancer location are shown in FIGURE2.

Radiotherapy in patients with cardiac implantable electronic devices Until the end of 2019, a total of 515 CIED patients before, during, or after radiotherapy were evaluated by the CO-CIED team. During 5872 radiotherapy sessions, 2 harmful interactions were recorded, and they are discussed in detail in a separate study. ${ }^{19}$ The number of patients with CIEDs per year, their baseline characteristics, and radiotherapy location are presented in FIGURE3.

Magnetic resonance imaging in patients with cardiac implantable electronic devices From January 2018 to December 2019, 48 MRI examinations were performed (18 in 2018 and 32 in 2019). All of them were performed safely, 


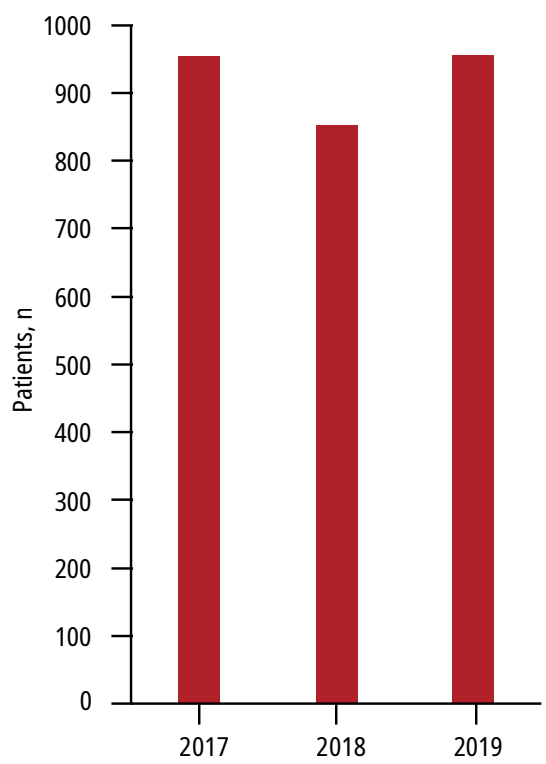

\section{Variable}

\begin{tabular}{ll} 
Age, y, mean (SD) & $62(12)$ \\
\hline Female sex, \% & 77 \\
\hline Outpatient consultations, \% & 61 \\
\hline In-hospital consultations, \% & 39 \\
\hline $\begin{array}{l}\text { Patients referred for an in- } \\
\text {-hospital CV evaluation before } \\
\text { cancer treatment initiation, \% }\end{array}$ & 1.1 \\
\hline $\begin{array}{l}\text { CV complications related to } \\
\text { cancer treatment, \% }\end{array}$ & 18 \\
\hline
\end{tabular}

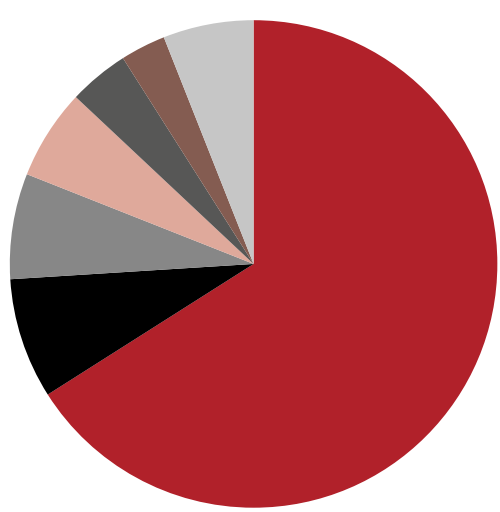

Others Lungs Head/neck Sarcoma GI Kidney Breast

FIGURE 2 The number per year (left), baseline characteristics (middle), and cancer location (right) of patients referred to the cardio-oncology service in Gliwice Abbreviations: CV, cardiovascular; GI, gastrointestinal

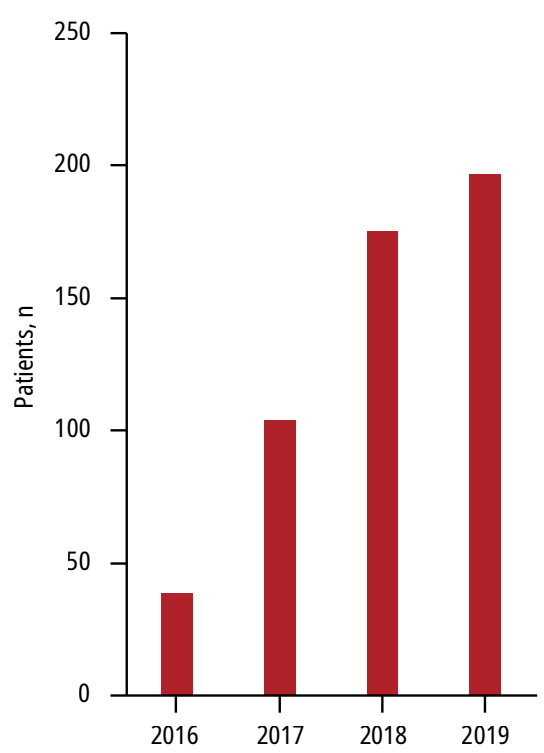

\begin{tabular}{ll} 
Variable & $\mathrm{n}=515$ \\
\hline Radiotherapy sessions, $\mathrm{n}$ & 5872 \\
\hline Age, $\mathrm{y}$, mean (SD) & $72(9)$ \\
\hline Male sex, \% & 78 \\
\hline Pacemaker, \% & 70.3 \\
\hline ICD, \% & 22.8 \\
\hline CRT-D, \% & 6.1 \\
\hline CRT-P, \% & 0.8 \\
\hline $\begin{array}{l}\text { Time from CIED implantation, } \\
\text { y, median (IQR) }\end{array}$ & $4.2(3.1)$ \\
\hline Pacemaker dependency, \% & 22.3 \\
\hline $\begin{array}{l}\text { Secondary prevention in } \\
\text { patients with ICD / CRT-D, \% }\end{array}$ & 33.3 \\
\hline Low risk, \% & 49.1 \\
\hline Medium risk, \% & 40.6 \\
\hline High risk, \% & 10.3 \\
\hline
\end{tabular}

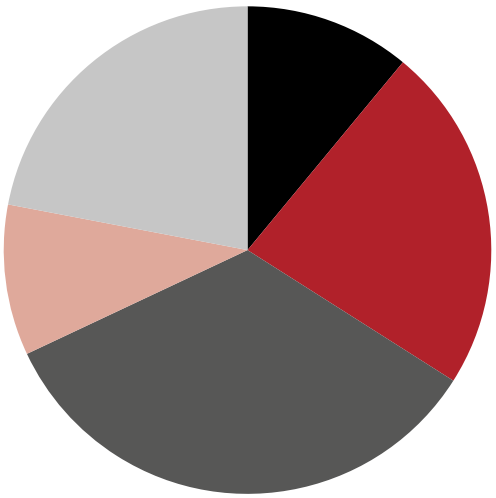

Others Head/neck Chest Pelvis Abdomen

FIGURE 3 The number per year (left), baseline characteristics (middle), and radiotherapy location (right) of patients undergoing radiotherapy at the cardio-oncology service in Gliwice

Abbreviations: CRT-D, cardiac resynchronization therapy-defibrillator; CRT-P, cardiac resynchronization therapy-pacemaker; DR, dual chamber; ICD, implantable cardioverter-defibrillator; others, see FIGURE 1

and no adverse events were recorded during and after MRI. No significant changes in battery voltage, impedance, pacing threshold, or sensing were observed. The baseline characteristics and the imaged area are presented in FIGURE 4.

Cancer surgery in patients with cardiac implantable electronic devices Until the end of 2019, cancer surgery in patients with cardiac implantable electronic devices was performed in 89 patients without any concomitant CIED-EMI complications.

DISCUSSION In the European Society of Cardiology position paper on cancer treatments and cardiovascular toxicity, ${ }^{10}$ cardiovascular complications of various cancer treatment modalities 


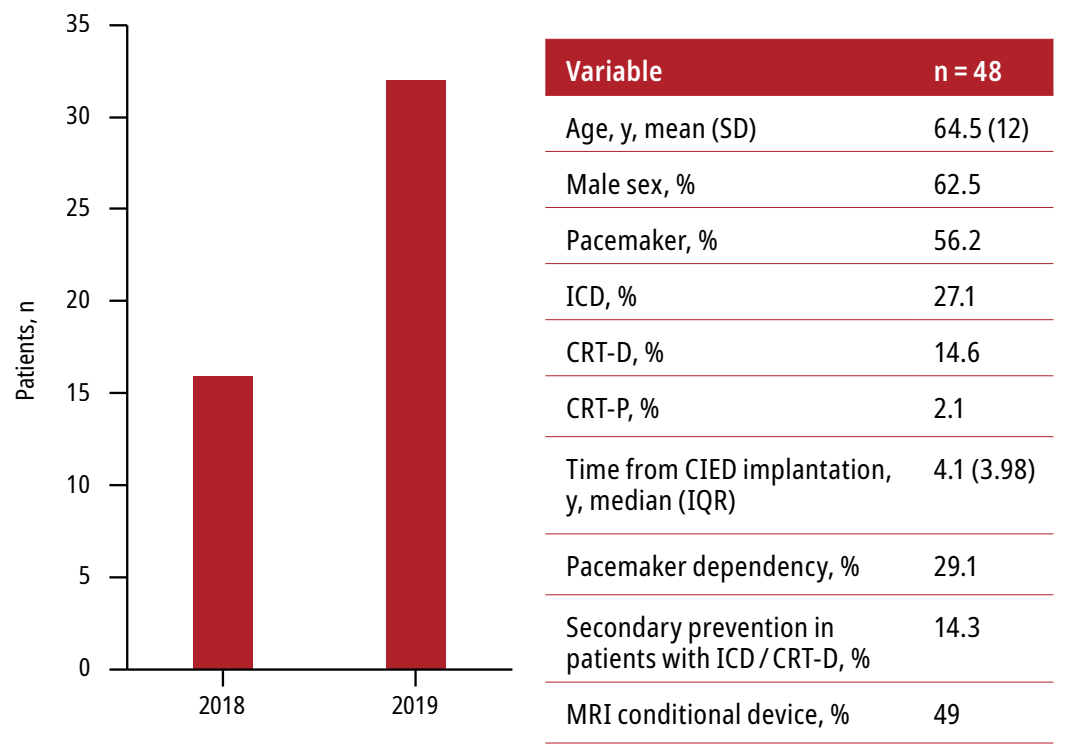

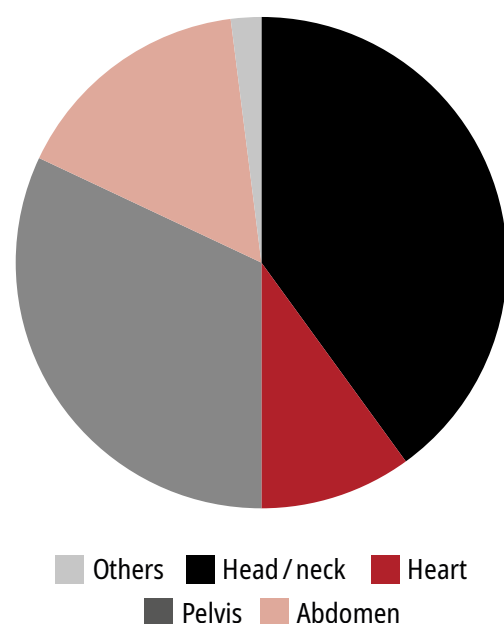

FIGURE 4 The number per year (left), baseline characteristics (middle), and location of magnetic resonance imaging (MRI; right) of patients undergoing MRI at the cardio-oncology service in Gliwice

Abbreviations: see FIGURE 3

were discussed and divided into 9 main categories: myocardial dysfunction, coronary artery disease, valvular disease, arrhythmias, arterial hypertension, thromboembolic disease, peripheral vascular disease, pulmonary hypertension, and pericardial complications. Consequently, the optimal, comprehensive management requires in-depth knowledge since it is the domain of 2 separate specialties. Additionally, in the majority of cases, there is a necessity to involve numerous physicians with an additional area of expertise in daily practice (within cardiology: specialists in heart failure, electrophysiology, etc.; within oncology: specialists in clinical oncology, radiotherapy, etc.) as well as experts in other related fields. Moreover, the exclusion of cancer patients from cardiological clinical trials and cardiovascular patients from oncological trials has resulted in the lack of data indispensable for direct clinical decision-making in many $\mathrm{CO}$ patients. Given that, it seems reasonable that a traditional care approach involving a cardiologist's and an oncologist's individual consultations with patients is not optimal to manage the joint cancer and cardiovascular needs, as it may result in fragmented care, variability, and delays in the assessment and clinical decisions, and subsequently in suboptimal outcomes..$^{20,21}$ Thus, a multidisciplinary-team approach to $\mathrm{CO}$ care is increasingly adopted in the current clinical practice worldwide. ${ }^{22}$

Recently, Snipelisky et $\mathrm{al}^{23}$ proposed 3 milestone solutions, each with 3 steps, as a road map to the successful implementation of a $\mathrm{CO}$ program (FIGURE 5). Although the presented $\mathrm{CO}$ service was developed earlier, some fundamental parts of the suggested road map were used. After a joint discussion with our colleagues, the initial vision of the $\mathrm{CO}$ service including a strong need for cooperation (mainly due to the size of the 2 included centers and the broad spectrum of possibilities in cardiovascular and cancer treatment) was assessed, along with objectives, location, team members, and perspectives. As a result, we obtained the support of both institutions.

Moreover, the implementation of an optimal $\mathrm{CO}$ program requires a dedicated environment. The organization of the $\mathrm{CO}$ service may vary depending on the localization, goals, the structure of the dedicated outpatient $\mathrm{CO}$ clinic, team members included, structured protocols and programs. These could depend on the hospital capacity and organizational structure, the availability of cardiovascular imaging, and the type/number of patients referred. Considering the above facts and being aware of the advantages and disadvantages of the establishment of a CO clinic within a cardiovascular rather than a cancer center, ${ }^{24}$ we decided that our $\mathrm{CO}$ clinic should be included in the oncology center. The $\mathrm{CO}$ clinic goals were defined and the CO team was established and launched its activity in 2016. We decided that the first line of our service will include only the outpatients. However, clinical indications may call for the inclusion of the inpatient line as combined with the cardiology center.

The population of patients with cardiac CIEDs is constantly growing. Notwithstanding HRS recommendations, which allow to perform MRI in this group of patients, CIEDs still remain an absolute contraindication in most of the Polish MRI laboratories. This may result in a disqualification from MRI, which is a valuable examination in 


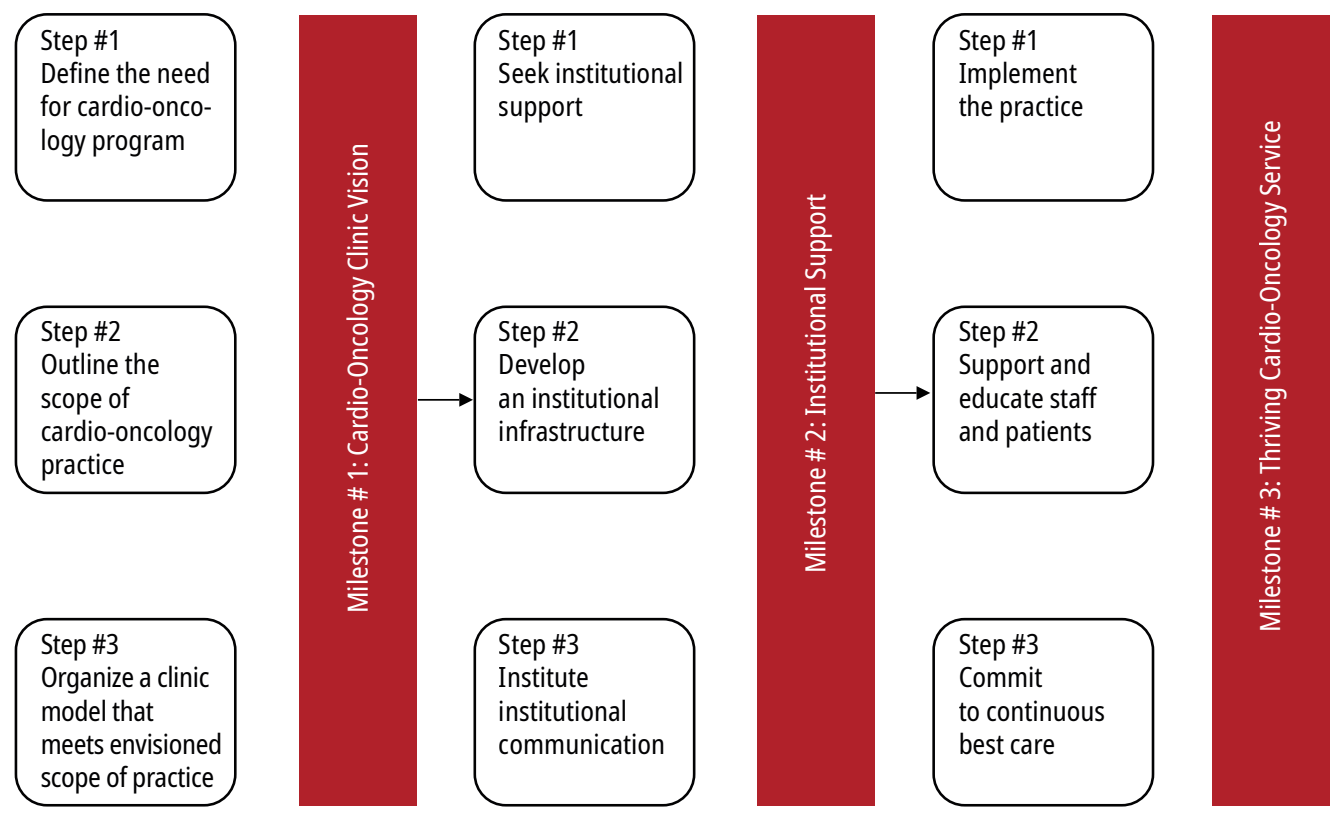

FIGURE 5 Key steps and milestones toward a successful cardio-oncology clinic. Reprinted from Snipelisky et al23 with permission from Elsevier.

patients with suspected cancer, as well as in patients already diagnosed with cancer and requiring a follow-up based on the MRI results. Therefore, we have developed this area of the CO service to make sure that all patients with CIEDs are eligible for MRI with appropriate precautions.

In the majority of cases, surgery remains one of the most important elements of cancer treatment. A growing number of these patients have CIEDs. Electromagnetic interference, which is mainly associated with the use of medical equipment in the operating theater (wireless technology, infusion pumps, monitoring devices, ultrasound probes, and diathermy) may affect the function of CIEDs. Therefore, a comprehensive evaluation including the control and reprogramming of CIEDs in patients scheduled for surgery guarantees a reduction in the number of complications and allows for a timely intervention if required.

Conclusions This report reveals baseline characteristics and initial clinical outcomes in one of the largest cohort of patients referred to the $\mathrm{CO}$ service established within the cancer center. Such a cooperation in evaluating cancer patients with regard to many potential interactions between cancer treatment and cardiovascular complications seems to be safe and feasible.

\section{ARTICLE INFORMATION}

CONTRIBUTION STATEMENT MT, IS, and AM were responsible for study design, data analysis, and manuscript preparation. SB, MJ, BB-B, EG-G, and MG revised the manuscript. MT and MG performed the final revision. All authors approved the final version of the manuscript.

CONFLICT OF INTEREST None declared.

OPEN ACCESS This is an Open Access article distributed under the terms of the Creative Commons Attribution-Non Commercial-No Derivatives 4.0
International License (CC BY-NC-ND 4.0), allowing third parties to download articles and share them with others, provided the original work is properly cited, not changed in any way, distributed under the same license, and used for noncommercial purposes only. For commercial use, please contact the journal office at kardiologiapolska@ptkardio.pl.

HOW TO CITE Tajstra M, Blamek S, Skoczylas I, et al. Two professions against two killer diseases: the rationale, organization, and initial experience of a cardio-oncology service. Kardiol Pol. 2021; 79: 139-146. doi:10.33963/KP.15674

\section{REFERENCES}

1 Causes of death statistics. Eurostat website. https://ec.europa.eu/eurostat/ statistics-explained/index.php/Causes_of_death_statistics. Updated September 20, 2019. Accessed September 12, 2020.

2 Thun MJ, DeLancey J0, Center MM, et al. The global burden of cancer: priorities for prevention. Carcinogenesis. 2010; 31: 100-110.

3 Eyre H, Kahn R, Robertson RM, et al; American Cancer Society, American Diabetes Association, American Heart Association. Preventing cancer, cardiovascular disease, and diabetes: a common agenda for the American Cancer Society, the American Diabetes Association, and the American Heart Association. Circulation. 2004; 109: 3244-3255.

4 Truong J, Yan AT, Cramarossa G, Chan KK. Chemotherapy-induced cardiotoxicity: detection, prevention, and management. Can J Cardiol. 2014; 30: 869-878.

5 Stowik AJ, Jagielski P, Potocki P, et al. Anthracycline-induced cardiotoxicity prevention with angiotensin-converting enzyme inhibitor ramipril in women with low-risk breast cancer: results of a prospective randomized study. Kardiol Pol. 2020; 78: 131-137.

6 Ewer MS, Ewer SM. Cardiotoxicity of anticancer treatments. Nat Rev Cardiol. 2015; 12: 620.

7 Barac A, Murtagh G, Carver JR, et al. Cardiovascular health of patients with cancer and cancer survivors: a roadmap to the next level. J Am Coll Cardiol. 2015; 65: 2739-2746.

8 Kalin R, Stanton MS. Current clinical issues for MRI scanning of pacemaker and defibrillator patients. Pacing Clin Electrophysiol. 2005; 28: 326-328.

9 Indik JH, Gimbel JR, Abe H, et al. 2017 HRS expert consensus statement on magnetic resonance imaging and radiation exposure in patients with cardiovascular implantable electronic devices. Heart Rhythm. 2017; 14: 97-153.

10 Zamorano JL, Lancellotti P, Rodriguez Mu noz D, et al. 2016 ESC position paper on cancer treatments and cardiovascular toxicity developed under the auspices of the ESC Committee for Practice Guidelines: The Task Force for cancer treatments and cardiovascular toxicity of the European Society of Cardiology (ESC). Eur Heart J. 2016; 37: 2768-2801.

11 Tarantini L, Gulizia MM, Di Lenarda A, et al. ANMCO/AIOM/AICO consensus document on clinical and management pathways of cardio-oncology: executive summary. Eur Heart J Suppl. 2017; 19: 370-379.

12 Gauter-Fleckenstein B, Israel CW, Dorenkamp M, et al. DEGRO/DGK guideline for radiotherapy in patients with cardiac implantable electronic devices. Strahlentherapie und Onkol. 2015; 191: 393-404. 
13 Hurkmans CW, Knegjens IL, Oei BS, et al. Management of radiation oncology patients with a pacemaker or ICD: A new comprehensive practical guideline in The Netherlands. Radiat Oncol. 2012; 7: 198.

14 Tajstra M, Gadula-Gacek E, Buchta $P$, et al. Effect of therapeutic ionizing radiation on implantable electronic devices: systematic review and practical guidance. J Cardiovasc Electrophysiol. 2016; 27: 1247-1251.

15 Blamek S, Gabrys D, Kulik R, et al. Stereotactic body radiosurgery, robotic radiosurgery and tomotherapy in patients with pacemakers and implantable cardioverters-defibrillators. Exp Clin Cardiol. 2014; 20: 757-763.

16 Indik JH, Gimbel JR, Abe H, et al. 2017 HRS expert consensus statement on magnetic resonance imaging and radiation exposure in patients with cardiovascular implantable electronic devices. Heart Rhythm. 2017; 14: 97-153.

17 Tajstra M, Blamek S, Niedziela JT, et al. Patients with cardiac implantable electronic devices undergoing radiotherapy in Poland. Expert opinion of the Heart Rhythm Section of the Polish Cardiac Society and the Polish Society of Radiation Oncology. Kardiol Pol. 2019; 77: 1106-1116.

18 Crossley GH, Poole JE, Rozner MA, et al. The Heart Rhythm Society (HRS) / American Society of Anesthesiologists (ASA) expert consensus statement on the perioperative management of patients with implantable defibrillators, pacemakers and arrhythmia monitors: facilities and patient management. Heart Rhythm. 2011; 8: 1114-1154.

19 Niedziela JT, Blamek S, Gadula-Gacek E, et al. Radiation therapy in patients with cardiac implantable electronic devices. Kardiol Pol. 2021; 79: 156-160.

20 Albini A, Pennesi G, Donatelli F, et al. Cardiotoxicity of anticancer drug: the need for cardio-oncology and cardio-oncological prevention. J Natl Cancer Inst. 2010; 102: 14-25.

21 Chen C, Steingart R. Cardiac disease and heart failure in cancer patients: is our training adequate to provide optimal care? Heart Fail Clin. 2011; 7: 357-362.

22 Pareek N, Cevallos J, Moliner P, et al. Activity and outcomes of a cardio-oncology service in the United Kingdom - a five year experience. Eur J Heart Fail. 2018; 20: 1721-1731.

23 Snipelisky D, Park JY, Lerman A, et al. How to develop a cardio-oncology clinic. Heart Fail Clin. 2017; 13: 347-359.

24 Okwuosa TM, Barac A. Burgeoning cardio-oncology programs: challenges and opportunities for early career cardiologists/faculty directors. I Am Coll Cardiol. 2015; 66: 1193-1197. 